\title{
The Study of Development Pattern of Micro-Blog Combined with E-commerce
}

\author{
Lei Liu \\ Dept. of information and engineering, Jilin business and technology college, Changchun, China, 130062 \\ Email: liuleiperfect@163.com
}

\begin{abstract}
In this paper, the author discussed the development pattern of Micro-blog combined with E-commerce. The feasibility of combination between both was analyzed. This paper pointed out e-commerce providing innovative profit model and development power to Micro-blog, and E-commerce enterprises can apply the platform of micro-blog to implement marketing and management. Additionally the problems and countermeasures which the combination brings were studied.
\end{abstract}

Keywords-Micro-blog; E-commerce; development pattern; mobile E-commerce; profit model

\section{INTRODUCTION}

E-commerce and Micro-blog are the two rapid development application of internet in China, they are influencing the people to apply the internet deeper level and changing the people's life style. With the development of IT in our country, rapid population of internet and the market state of incomplete retailing service, there's more development rooms for E-commerce. Especially, the problems of security and trust such as e-payment were solved well, E-commerce made a great progress. With the Sina Micro-blog setting off the tide like Twitter in the year of 2009, the Chinese internet began to enter a whole era of microblogging. It has remarkable features: short content, timeliness, spread quickly, more announcing channels and strong interactivity etc. It meets the people's demands of expressing personal emotion and understanding concerned persons and things in our fast-paced life.

Rapid development Micro-blog is in urgent need of profit model and further development power, at the same time, E-commerce need a new development platform. It is inevitable to combine two together for internet application service. But it is still in the stage of exploration. Whether the combination of both can provide the attractive and convenient service for user or not? Can it make commercial value for Micro-blog? Whether it can develop the new model for E-commerce? What the problems it probably brings?

\section{COMBINATION BASIS: WELL DEVELOPMENT STATE}

\section{A. continued stable growth E-commerce}

According to recent statistics from iResearch, in the second quarter of 2011, the scale of E-commerce industry trade is 1.6 trillion yuan. iResearch forecasts the scale of E-commerce industry trade reaches 13.9 trillion yuan until
2013, compound growth rate of each year is $42.6 \%$, with increasing progressively year by year. Deutsche Bank pointed out in September 2010 that personal consumption E-commerce market including $\mathrm{B} 2 \mathrm{C}$ and $\mathrm{C} 2 \mathrm{C}$, compound growth rate will reach $42 \%$, and $7.2 \%$ of the total gross domestic retail. The number of consumers who go network shopping 1.5 billion. Additionally, much data showed consumer groups who use mobile phone to shop online is expanding ceaselessly, mobile payment also began to grow. China Internet Network Information Center published "The twenty-ninth statistical report on Internet development in China”. The report showed that Internet users in China exceeded 500 million as of the end of December 2011. Internet penetration rate was increased four percentage points compared with 2010. Chinese mobile phone users reached 356 million, an increase of 17.5 percent compared to previous years, it has become an important part of Chinese Internet users. So, the growth of Chinese overall netizens comes into the plateau. The proportion of mobile phone users to use Micro-blog was from $15.5 \%$ in late 2010 to $34 \%$. This is a powerful source of power for E-commerce and mobile E-commerce development. On Taobao now more than 200,000 businesses have opened phone mobile shop.

As E-commerce is the connection point of the virtual world and the real world, and need to have the logistics, capital flow, information flow, so it involves a lot of problems and the strongest challenge too. In order to achieve new progress in the future and find the way of sustained profitability, so it is necessary to integrate effectively with new Internet applications.

\section{B. The Micro-blog explosive development}

Now in China the most widely used Micro- blog sites include Sina and Tencent.

Sina which at first launched Micro-blog service in August 2009 attracted 50 million users within a year, while the Twitter won the number of users with three years Sina's latest statistical data showed that Sina Microblog registered users has exceeded 300 million, the number of users is more than 100 million daily. The world-renowned market research company Nielsen released a report, it showed that now the users of Sina Micro-blog are more active than those of Micro-blog ancestor Twitter.

Referring to Tencent Micro-blog, the number of registered users exceeded 373 million till the end of last year, now over 400 million, the number of active users reaches 68 million. 
Whether the scale of users, or user increment rate, Micro-blog business development is very rapidly, a large number of users are gathered in the short term, resulting in Micro-blog has gradually become a standard to lots of Internet companies.

The commercial value inherent in the super popularity tapped and turned the commercial interests, that is, how to profit and how to set up valuable business model. It is the puzzle of Micro-blog facing.

Experience has shown that the more participants, the more the market exists, the more business opportunities in the Internet application. E-commerce has been relatively mature service delivery model, and Micro-blog condenses a lot of user groups, they both have good user service foundation respectively, If you could find an entry point for a combination of both, and put forward supporting service mechanism, not only it can greatly enhance the user experience feelings of information, but also provide a new profit model for Micro-blog service, at the same time, the market gains of E-commerce service providers are increased, so to form win-win closely aligned with the momentum of development.

\section{THE VALUE OF A COMBINATION OF BOTH}

\section{A. The value of E-commerce to the Micro-blog}

Firstly, integrating the development mode of E-commerce, enriching information services types, enhancing user experience, maintaining the user's the microblogging viscosity, reducing the loss of users.

Currently main operation mode of Micro-blog is expressing their personal emotional life, tracking characters and events information. This service allows users to release and share short message quickly and timely, so lots of users were gathered in short time quickly. Single service business will probably be taken placed in the market competition. Throughout the network development process, we can see that many new business can condense quickly lots of users at the first development stage due to users' curiosity. But with users' enthusiasm drop, or the appearance of newer business, the number of users will be decreased greatly etc. All factors show that it is difficult to keep long-term stable development for any lacking of innovative new business. When Micro-blog service is of great concern, we can provide related E-commerce business at the right time to continuously enhance the user stickiness.

Secondly, directly carry out profit models of E-commerce, excavate big commercial value of Micro-blog. It is a key to whether can exist long-term for Micro-blog. Comprehensive view, several aspects as follow:

\section{(1) Advertising revenue}

Setting up big platform, relying on high click rate can attract brand advertisers. Specific forms of advertising: product placement, sponsored advertising and keyword advertising. For product placement, the way of Twitter: to allow individual users to gain profit through inserting advertising into the personal page. Users and advertisers determine advertising time and fees through consultation,
Twitter take 5\% service charge, users' gains are deposited in the virtual account set by Twitter advertising department in accordance with the agreed ratio and broadcast time, after the advertising finished, the money can be transferred to the user's real account. Twitter keyword advertising was a success. Twitter officially launched the advertising program: Promoted Tweets. In advertising platform, business users can purchase specific keywords, when users search the keyword relevant ads are displayed.

\section{(2) To create open Micro-blog platform}

We can adopt the mode of win-win cooperation between the developers and the Micro-blog platform. Among the lessons to be learned that new business model: Apple pioneered the integration of Internet and mobile phone. That is APPStore. On the platform, about 80\% applications need to pay fee which are divided into 3:7 between Apple and the developer. So, through this platform, tens of thousands of developers can join in, so as to we finish seamless interconnection with other blog, Micro-blog and SNS site and strength the users' stickness, make profit finally.

\section{(3) Carving up profits with telecom operators}

With 3G official business, mobile media come into rapid development period, various APIs make users login Micro-blog through mobile phone. So, Micro-blog platform can carve up web traffic and SMS with telecom operators. For example, most of the traffic of Twitter come from the phone the mobile Internet and SMS.

(4) Combined with existing E-commerce models to develop innovative Micro-blog products. It is the biggest advantage that Micro-blog never lack of web traffic. Learned from existing profit model of group purchasing website and similar Taobao we can build a micro-group buying and micro-shop. If through carrying out activity such as "seckill", group buying on Micro-blog to sell economical, affordable products, with the addition of opinion leader's promotion and call, it is bound to achieve great economic and social benefits, so that win-win situation are formed.

(5) Charging for business users. To build brand ID Mall and obtain profit by certification, admission fees and charge a brokerage commission. Business users also can take the traffic analysis tools, in order to develop more potential customers.

\section{(6) Providing valuable data and information}

In Micro-blog, the data of users and information form massive information base, they are worth mining deeply. For example, the demands of consumers for different brands and different products, the evaluation of brand and product are recorded and assessed to form related monitoring, in order to provide a tool for business knowing users' demands and brand reputation. That is providing paid information. Dell pays the network monitoring company Visible Technologies on Twitter to grasp its user feedback on the company's products, and have achieved a certain economic benefits.

(7) Through the so-called value-added services to gain profit. To release a series of diversity, individuality, and utility payment services for users and constantly improve Microblog function. 


\section{B. The value of Micro-blog to E-commerce}

The online social + E-commerce has become one of the most popular topic. For business, the biggest value in use of Micro-blog is enterprises promotion that is Micro-blog marketing. As the Microblog is easy-to-use, as well as the influence of the interpersonal circle, Micro-blog has a great advantage. Precise marketing channels can reduce marketing costs, reduce the risk of the proliferation of advertising leading to users producing aversion and the bad image of your product. If promotion is successful will produce good results.

Micro-blog marketing is usually the following ways:

(1) The platform of enterprise releasing information and transmitting culture

Dell began to use Twitter platform to publish information from March 2007: Company news, discount information, blog account, community account. The principle of publishing information is firstly, any information must be valuable; secondly, feedback must be timely and interactive. Enterprise can initiate marketing activities. There are many such success cases. Publishing various valuable information not only quickly gather loyal and potential customer, but also express enterprise brand concepts and brand culture imperceptibly.

(2) Advertising. In personal Micro-blog, product placement can give expression to the opinions of product and service. This marketing means can be used in celebrity Micro-blog. It has good marketing effects.

(3) Fast customer service channel. Some enterprise made the official Micro-blog as the platform of communicating with customer and after-sale service. Through the platform the enterprise can quickly know about customer's doubt and demand and timely reply them by Micro-blog, e-mail or telephone etc. It can effectively improve the satisfaction of customer.

(4) A platform that the enterprise understands the customer deeply. Micro-blog is a effective platform that the enterprise can better listen, study and understand the customer. The blogger recorded the real idea, hobby, demand, plan and thoughts and so on, really expressed their consumption needs, predilection, life style and brand attitude, especially purchase channels, purchase consideration factors. It is helpful to understand customer deeply in order to develop or optimize product strategy and marketing strategy.

(5) A platform of reputation monitoring and crisis management. Micro-blog fission and explosive spread can result in enterprise falling into public relations crisis quickly due to the negative comments of some products or services and the negative evaluation of the brand. Micro-blog is a timely and important platform on which the enterprises carry out crisis management, indicate their attitude and position. On Micro-blog the enterprise's official blog and enterprise president can communicate with netizens face to face sincerely to ease the negative public opinion. So the Micro-blog platform can timely monitor the public praise.

Compared with personal Micro-blog, has unique value. This business' Micro-blog is closed. It is taken as internal communication platform. Its value lies in the enterprise information management and collaborative office. There do not exist the website that focuses on this market in China.

\section{The combination of Micro-blog and E-commerce enterprises promote the development of mobile E-commerce}

Mobile E-commerce is E-commerce activities which are use of mobile phones, PDAs and handheld computers and other wireless terminals. Mobile E-commerce will be E-commerce future development trend with the characters of open, everywhere, anytime, anywhere and so on. With the appearance of application innovation and new technology and multilateral participation such as telecom operators, financial sector, the traditional channels, equipment manufacturers, emerging E-commerce providers, business model of mobile E-commerce will be changed greatly. All partners make efforts to achieve complementary advantages, and win-win cooperation. For the industrial chain of mobile E-commerce, Micro-blog offers a large number of users, ensure information timeliness, convenience, and the effectiveness of business information; under the premise of ensuring the number of users, E-commerce businesses will be attracted to the mobile phone platform.

\section{EXISTED PROBLEMS AND COUNTERMEASURES}

Profit is the ultimate goal of any kind of business model. As far as E-commerce and Micro-blog, their respective the advantages generate the forces and impact as we all know, but when it comes a combination of both to achieve profit the purpose of, its potential has not yet been completely released out. Internet industry and the E-commerce businesses must still maintain a clear understanding in the face of rapid development and huge potential market opportunities.

Firstly, with knowing about Micro-blog, people's enthusiasm may be decreased, the regular user quantity of and stickness would be probably decreased.

Secondly, from the entire social network, the total market capacity has a development limits, it is difficult to keep absolute superiority in the user quantity.

Thirdly, Micro-blog will bear the great risk of policy factors. Dispute also would be great on powerful Micro-blog. As media feature, the content is very difficult to control; as social interaction feature, higher requirements on the identification of the user identity and privacy protection are put forward. The enterprises should have strong strength to apply this developing platform.

Lastly, From the point of view of the current domestic and international situation, applying Micro-blog marketing is suitable for all companies. Some big companies do it better, because they own very strong professional team, abundant capital, and the supports with celebrity or opinion leader on Micro-blog. So, there are many difficulties for medium-sized and small enterprises to make Micro-blog marketing.

\section{THE END}

The potential of social intercourse Micro-blog do not yet completely release, it will open up the pattern of social intercourse E-commerce. 
Though there still exists some problems for the business pattern of combination of the two, with the rapid development of network technology and ceaseless mature, the organic and reasonable combination of the two will be a new potential outcome.

\section{REFERENCES}

[1] China Internet Network Information Center: The 29th China Internet Network Development Report, 2012.01.16. http://www.cnnic.cn/research/bgxz/tjbg/201201/t20120116_23668.ht $\mathrm{ml}$

[2] Meihn Zhao. The Prospect of Sina Micro-blog[J]. Journalism \& Communication, 2011,3:74-75.
[3] Xuan Zhou. Analysis of New E-commerce Model by Micro-blog Media[J]. Modern Business Trade Industry, 2011,14:222-223.

[4] Er Xie, Wenke Ren. The Power of Micro-blog[M]. Renmin University of China Press, 2010

[5] Lepeng Wang, Chunli Li, Ying Wang. Study on Necessity and Strategy of SNS Website Combined with E-commerce[J]. Market Forum, 2010,11:58-59.

[6] Neri Mario Arrigoni, Colombetti Marco. Ontology-based Learning Objects Search and CoursesGeneration. Applied Artificial Intelligence, 2009,23(3):233-260.

[7] Qingyan Li; Duo Jin; Wenan Zhang; Tao Zhang Research on the Development Trend of M-Commerce[J]. Telecommunications Science, 2011,06: 6-13. 